\title{
Effects of Exogenous Melatonin on Photosynthetic Characteristics of Capsella bursa-pastoris Seedlings under Salt Stress
}

\author{
Juan $\mathrm{He}^{1, \mathrm{a}}$, Guochao Sun ${ }^{2, \mathrm{~b}}$ and Yi Tang ${ }^{2, \mathrm{c*}}$ \\ ${ }^{1}$ College of Horticulture, Sichuan Agricultural University, Chengdu, Sichuan, China \\ ${ }^{2}$ Institute of Pomology and Olericulture, Sichuan Agricultural University, Chengdu, Sichuan, China \\ a1226246433@qq.com, bsunguochao@163.com, c95459425@qq.com
}

${ }^{*}$ Corresponding author. Juan He and Guochao Sun contributed equally to this work.

\begin{abstract}
Keywords: Capsella bursa-pastoris; Melatonin; Salt stress; Photosynthetic characteristics Abstract: A pot experiment was conducted to study the effects of spraying with 5 different concentrations $\left(0,50,100,150,200 \mu \mathrm{mol} \cdot \mathrm{L}^{-1}\right)$ of exogenous melatonin (MT) on photosynthetic characteristics of $C$. bursa-pastoris seedlings under $50 \mathrm{mmol} \cdot \mathrm{L}^{-1} \mathrm{NaCl}$ stress. The results showed that spraying exogenous MT significantly increased the net photosynthetic rate $(\mathrm{Pn})$, transpiration rate $(\mathrm{Tr})$ and stomatal conductance (GS) of C. bursa-pastoris seedlings and all increased at first then decreased. The changes trend of the intercellular $\mathrm{CO}_{2}$ concentration $(\mathrm{Ci})$ in the C. bursa-pastoris seedlings were contrary to that of $\mathrm{Pn}, \mathrm{Tr}$ and Gs, and decreased with the increase of MT concentration. Therefore, spraying exogenous MT can improve the photosynthetic capacity of $C$. bursa-pastoris seedlings, which will help to improve the adaptability of $C$. bursa-pastoris, and the best concentration is 150 $\mu \mathrm{mol} \cdot \mathrm{L}^{-1}$.
\end{abstract}

\section{Introduction}

Capsella bursa-pastoris is a one-year or biennial herbaceous plant of Cruciferae. It's distributed in the temperate and subtropical regions of the southern hemisphere, and distributed throughout China and cultivated artificially. C. bursa-pastoris whole body is treasure, edible nutrient-rich, medicinal function as much. It also has the function of eyesight, hemostasis, liver gas, killing all the poison [1, 2]. C. bursa-pastoris contains a variety of carbohydrates, alkaloids, flavonoids, organic acids, peptides and other bioactive substances, is a highly developmental semi-wild resources [3].

In recent years, as soil salinization becomes more and more serious, the growth of plants is restrained. The growth of plants is slow and the metabolism is inhibited which resulting the yield and quality of crops are reduced, and in severe cases the plants wilt or even die [4-6].

The melatonin (MT, N-acetyl-5-methoxytryptamine), found in 1958 by Lerner in bovine pineal gland, is a hormone that widely exists in animals and plants and is used to regulate circadian rhythms [7]. MT can also help to accomplish a variety of physiological functions in plants. It can promote seed germination, stimulate root growth, regulate photoperiod, protect chlorophyll and delay leaf senescence that similar growth regulation effect to IAA [8]. Some studies show that, exogenous MT can improve plant stress resistance by inhibiting the degradation of photosynthetic pigments, increasing the light rate and enhancing the gene expression of antioxidants, and reducing the oxidative damage caused by salt stress [9-11]. Other scholars found that, the application of $1 \mu \mathrm{mol} \cdot \mathrm{L}^{-1}$ MT to Begonia cambogia can effectively increase the growth of Hubei begonia under salt stress and reduce the chlorophyll degradation and increase the photosynthetic rate [12]. In recent years, MT has become more and more widely used in alleviating plant stress and has become a current research craze. However, there is no report on the effects of MT on the photosynthetic characteristics of $C$. bursa-pastoris under salt stress. Therefore, in this study, C. bursa-pastoris was used as material to study the effects of exogenous MT on photosynthetic characteristics of $C$. bursa-pastoris under salt stress. 


\section{Materials and Methods}

Materials. C. bursa-pastoris seedlings were collected from Chengdu, Sichuan Agricultural University campus surrounding farmland. MT is purchased from SIGMA ALDRICH company, a small amount of anhydrous ethanol dissolves with distilled water as $500 \mu \mathrm{mol} \cdot \mathrm{L}^{-1}$ mother liquor, $4^{\circ} \mathrm{C}$ preservation, according to the design concentration dilution. All chemicals used in experiments were of analytical grade.

Experimental Design. Clean the $C$. bursa-pastoris seedlings, select growing evenly seedlings and transplant to pots which filled with substrate and the size is $21 \mathrm{~cm} \times 20 \mathrm{~cm}$ (diameter $\times$ height), then put the pots into plastic greenhous, growing under natural conditions and watering $20 \mathrm{~mL} 1 / 2$ Hoagland nutrient solution every other day. After C. bursa-pastoris seedlings transplanting survival, water Hoagland nutrient solution which containing $50 \mathrm{mmol} \cdot \mathrm{L}^{-1} \mathrm{NaCl}$ until the end of the trial in order to prevent water shortages, according to the weather conditions add moisture properly. When 4 true leaves of $C$. bursa-pastoris fully expanded, choose the same size of $C$. bursa-pastoris seedlings to spray 5 different concentrations of MT $\left(0,50,100,150,200 \mu \mathrm{mol} \cdot \mathrm{L}^{-1}\right)$ on leaves at 17:00 every other day, a total of 3 times.

30 days after intercropping, the photosynthesis of each plant was determined by using LI-6400 portable photosynthesis meter (LI-COR Inc., USA). The photosynthetic parameters of the photosynthesis meter were manual control $\mathrm{CO}_{2}$ concentration $400 \mu \mathrm{mol} \cdot \mathrm{CO}_{2} \mathrm{~mol}^{-1}$, temperature $25^{\circ} \mathrm{C}$, light intensity $1200 \mu \mathrm{mol} \cdot \mathrm{m}^{-2} \cdot \mathrm{s}^{-1}$. The determination of photosynthetic parameters were net photosynthetic rate $(\mathrm{Pn})$, transpiration rate $(\mathrm{Tr})$, stomatal conductance $(\mathrm{Gs})$ and $\mathrm{CO}_{2}$ concentration of intercellular $(\mathrm{Ci})$, and each treatment was repeated three times.

Statistic analyses. Statistical analyses were performed using SPSS 13.0 statistical software (IBM, Chicago, IL, USA). Data were analyzed by one-way ANOVA with least significant difference (LSD) at a $5 \%$ confidence level.

\section{Results and Discussion}

Net Photosynthetic Rate (Pn). Compared with the control, spraying exogenous melatonin under salt stress significantly increased the Pn of C. bursa-pastoris (Fig. 1). The Pn of C. bursa-pastoris firstly increased and then decreased with the increase of MT concentration, which was the highest at the MT concentration of $150 \mu \mathrm{mol} \cdot \mathrm{L}^{-1}$ and increased by $81.81 \%$ compared with the control. It decreased slightly at the MT concentration of $200 \mu \mathrm{mol} \cdot \mathrm{L}^{-1}$, but it was still significantly higher than the control, increased $63.50 \%$ compared with the control.

Transpiration Rate (Tr). With the Tr of C. bursa-pastoris, the trend was the same as that of Pn, and the Tr of $C$. bursa-pastoris was significantly higher than that of the control after exogenous MT and the trend was increased first and then decreased with the increase of MT concentration (Fig. 2). When the concentration of MT was $150 \mu \mathrm{mol} \cdot \mathrm{L}^{-1}$, it got the highest, which increased $109.77 \%$ compared with the control. It shows that exogenous melatonin can effectively improve the $\operatorname{Tr}$ of $C$. bursa-pastoris seedlings in a certain concentration range.

Stomatal Conductance(Gs). The Gs of $C$. bursa-pastoris seedlings after spraying different concentrations of exogenous melatonin under salt stress the Gs was ranked as: $150 \mu \mathrm{mol} \cdot \mathrm{L}^{-1}>200$ $\mu \mathrm{mol} \cdot \mathrm{L}^{-1}>100 \mu \mathrm{mol} \cdot \mathrm{L}^{-1}>50 \mu \mathrm{mol} \cdot \mathrm{L}^{-1}>0 \mu \mathrm{mol} \cdot \mathrm{L}^{-1}$ (Fig. 3). The same trend as that of $\mathrm{Pn}$ and $\mathrm{Tr}$, all showed a trend of first rising and then decreasing, and reaching the highest when the concentration of melatonin was $150 \mu \mathrm{mol} \cdot \mathrm{L}^{-1}$. Compared with the control, Gs increased $24.83 \%, 75.88 \%, 154.15 \%$ and $137.58 \%$ respectively after spraying melatonin.

Intercellular $\mathrm{CO}_{2}$ Concentration(Ci). The trend of $\mathrm{Ci}$ in C. bursa-pastoris seedlings was opposite to that of $\mathrm{Pn}, \mathrm{Tr}$ and Gs, the tendency to decrease first and then increase with the increase of MT concentration (Fig. 4). When the concentration of melatonin was $150 \mu \mathrm{mol} \cdot \mathrm{L}^{-1}$, it was $35.65 \%$ lower than that of the control. 


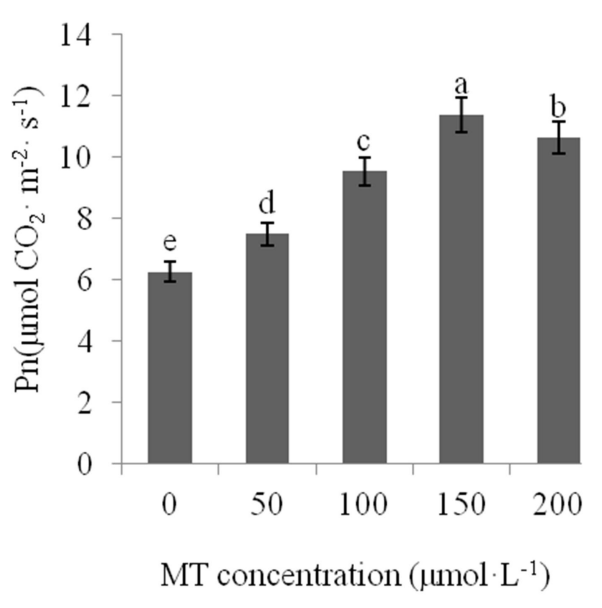

Fig. 1 Pn of C. bursa-pastoris seedlings

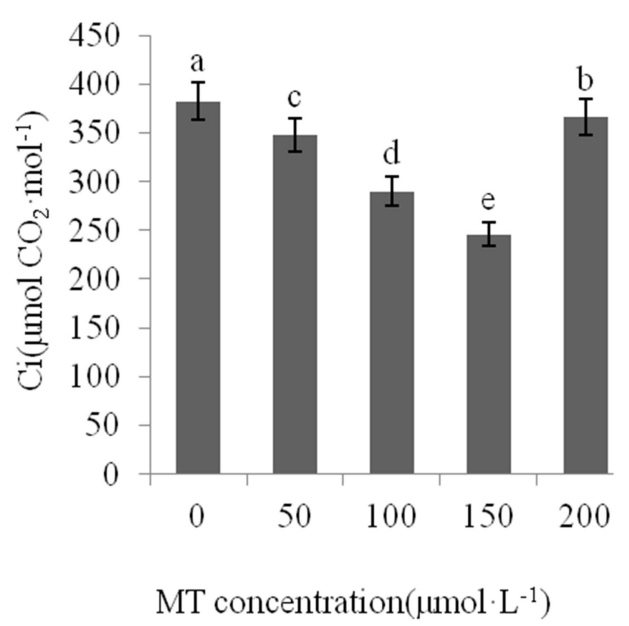

Fig. $4 \mathrm{Ci}$ of C. bursa-pastoris seedlings

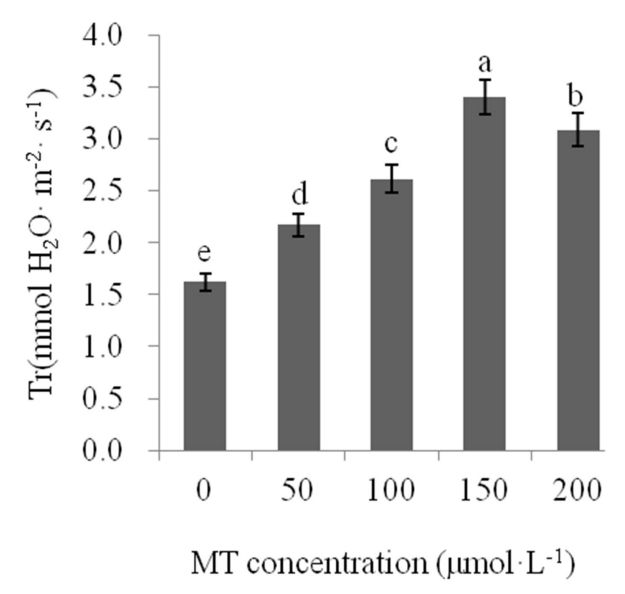

Fig. 2 Tr of C. bursa-pastoris seedlings

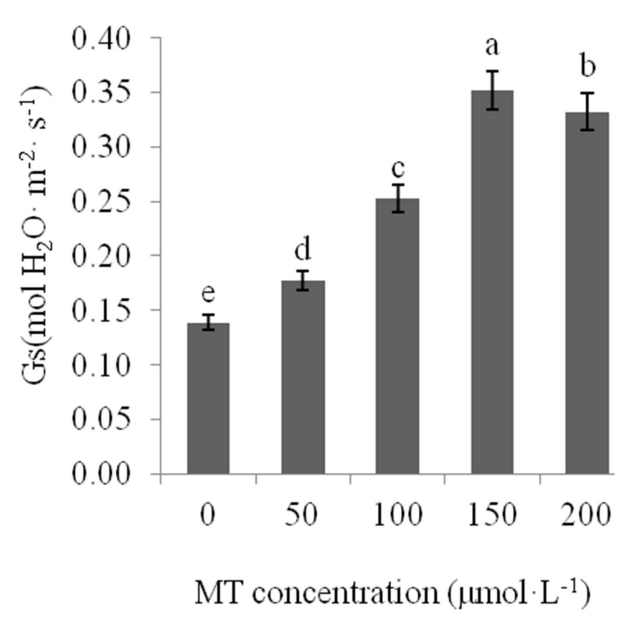

Fig. 3 Gs of C. bursa-pastoris seedlings

\section{Conclusions}

Photosynthetic pigment content in plant leaves directly reflects the photosynthetic capacity of plants, salt stress leads to the degradation of photosynthetic pigments in plant leaves and the breakdown of chloroplast fine structures, which hinders the photosynthesis of plants. Carbohydrates required for plant cell growth are mainly produced by photosynthesis, and high concentrations of salt stress also inhibit plant photosynthesis. MT by protecting the integrity of chlorophyll to maintain high photosynthetic rate. The results showed that, after spraying low concentraion of MT, Tr increased, which indicated that MT could promote plant to decrease leaf surface temperature and maintain higher enzyme activity through enhancing transpiration, thereby indirectly improving photosynthetic rate. Gs increased and $\mathrm{Ci}$ decreased, indicating that low concentration of MT increased stomatal conductance and reduced stomatal factors to inhibit photosynthesis, while enhanced $\mathrm{CO}_{2}$ fixation and transformation efficiency, and reduced the effect of non stomatal factors. Therefore, spraying exogenous of melatonin can effectively improve the photosynthesis of $C$. bursa-pastoris seedlings and promote the growth of $C$. bursa-pastoris seedling. The effect of spraying $150 \mu \mathrm{mol} \cdot \mathrm{L}^{-1}$ on MT was the best. 


\section{Acknowledgements}

This work was financially supported by the Application Infrastructure Project of Science and Technology Department of Sichuan Province (2016JY0258).

\section{References}

[1] H. Liang, X.Z. Zhang and J.H. Wang: Medical Journal of Qilu. Vol. 9 (1994), p. 123.

[2] A.F. Cheng: Vegetables. Vol. 6 (2004), p. 36.

[3] Z.H. Li and Y.X. Yao: Chinese Wild Plant Resources. Vol. 19 (2000), p 41.

[4] D. Bartels and R. Sunkar: Critical Reviews in Plant Sciences. Vol. 24 (2005), p 23.

[5] Y. Mei, Y.X. Zu and Y.C. Wu: Chinese Agricultural Science Bulletin. Vol. 31 (2015), p 49.

[6] S.W. Lu, F. Qi and T.L. Li: Northern Horticulture. Vol. 9 (2012), p 14.

[7] J.Q. Zuo, J.H. Xie and Y.X. Xue: Genomics and Applied Biology. Vol. 33 (2014), p 709.

[8] P. Wang, L. Yin and D. Liang: Journal of Pineal Research. Vol. 53 (2012), p 11.

[9] H.M. Li and Y. He: Acta Agriculturae Boreali-occidentalis Sinica. Vol. 22 (2013), p 131.

[10]X.D. Xu, Y. Sun and X.Q. Guo: Journal of Nuclear Agricultural Sciences. Vol. 25 (2011) p 179.

[11]L. Sun, Y.F. Zhou and F.X. Li: Scientia Agricultura Sinica.Vol.45 (2012), p 3265.

[12]C. Li, P. Wang and Z. Wei: Journal of Pineal Research. Vol. 53 (2012), p 298. 